\title{
Educación Sexual Integral: reflexiones sobre experiencias institucionales en el nivel secundario ${ }^{1}$
}

\author{
( María Soledad Vázquez \\ Universidad de Buenos Aires, Argentina \\ vazquezmariasoledad1974@gmail.com
}

\begin{abstract}
Resumen
En este artículo pongo en diálogo diferentes experiencias de Educación Sexual que tuvieron lugar en el nivel secundario de una institución de gestión privada con otras desarrolladas en un Bachillerato Popular. En la primera, se escolarizan adolescentes de entre 12 y 18 años que, en su mayoría, son de sectores socioeconómicos medios y medios-altos; en la segunda, jóvenes y adultos que atraviesan profundas vulnerabilidades materiales y simbólicas. Ambas escuelas son laicas y se ubican en diferentes municipios de la provincia de Buenos Aires.
\end{abstract}

Se trata de experiencias sucedidas en los últimos cuatro años, a las que accedo desarrollando dos roles diferentes: en la escuela de gestión privada, como docente orientadora con tareas de Educación Sexual Integral a cargo; en el Bachillerato Popular, como investigadora, en el marco del trabajo de campo de mi tesis doctoral.

Mi tesis está centrada en las escolaridades y en los vínculos que ocho jóvenes con experiencias de maternidad y de sectores populares sostienen con sus propias madres. Su metodología es el enfoque biográfico. Actualmente, estoy abocada a su escritura.

En ambas instituciones, ya sea como docente orientadora o como investigadora, he visualizado que varios estudiantes y sus familias rechazan la Educación Sexual Integral, sobre todo el tratamiento de dos de sus dimensiones claves: el respeto por la diversidad y el ejercicio de los derechos. Sin embargo, los conflictos asociados a estos rechazos son abordados institucionalmente de diferentes modos. Como desarrollo en este artículo, en la escuela de gestión privada la conducción directiva procura evitar cualquier tipo de controversia silenciando las temáticas rechazadas. En el Bachillerato Popular, en cambio, se consideran los conflictos como parte de las condiciones de abordaje de la sexualidad en la escuela y, desde allí, se habilitan espacios pedagógicos para su despliegue.

Palabras clave: Educación Sexual Integral, secundaria, sexualidades

1 Agradezco al director de mi tesis doctoral, Dr. Pablo Di Leo, y al equipo de investigación del que formo parte desde hace una década por su apoyo para la escritura de este artículo. 


\title{
Integral Sexual Education: thinking about institutional experiences in High School
}

\begin{abstract}
In this article, I put in dialogue different experiences of Sexual Education that took place in two different High Schools. One of these was in a private management institution while the other one was in a Popular High School. In the first school, there are teenagers between the age of 12 and 18, most of them from middle and upper-middle socio-economic sectors. In the second school, some young people and adults go through deep material and symbolic vulnerabilities. Both schools are secular and are located in different municipalities of the Province of Buenos Aires.
\end{abstract}

These experiences have happened in the last four years. I was able to access in these schools by developing two different roles. In the private management school as a guidance teacher with Integral Sexual Education tasks in charge. In the Popular High School, as a researcher during the fieldwork of my doctoral thesis.

My thesis is focused on scholarship and the relations that eight young people, which has experiences of motherhood and from popular sectors, have with their mothers. Its methodology is the biographical approach. Currently, I am writing my research. In both institutions, I have seen as a guidance teacher and as a researcher, that several students and their families reject Integral Sex Education, especially the treatment of two of its main dimensions: respect of diversity and the exercise of respect sexual rights. However, the conflicts associated with these rejections are addressed institutionally in different ways. In the private management school, the directive conduction tries to avoid any type of controversy by silencing the rejected topics. In the Popular High School, conflicts are considered as part of the conditions of teaching sexuality in school and, from there, pedagogical spaces are enabled for deployment.

Keywords: Integral Sexual Education, High School, sexualities

\section{Introducción}

En 2006 se sancionó la Ley Nacional 26.150 de Educación Sexual Integral, una normativa que crea política pública -el Programa Nacional de Educación Sexual Integral (en adelante ESI)- asignando recursos para su despliegue.

A partir de allí, se desarrollaron y se desarrollan varias líneas de acción para su efectivización, como por ejemplo la elaboración de los lineamientos curriculares (aprobados en 2008 por el Consejo Federal de Educación), la producción de materiales didácticos, e instancias de capacitación destinadas al colectivo docente.

Dicha normativa establece que todxs lxs educandxs² tienen derecho a recibir ESI, una práctica pedagógica centrada en las sexualidades, basada en conocimientos científicamente validados, a desarrollar de manera sistemática considerando, por un lado, 
aspectos biológicos, psicológicos, sociales, afectivos y éticos, y, por otro, la perspectiva de género, el cuidado del cuerpo y la salud, el respeto por la diversidad, el ejercicio de los derechos y la valoración de la afectividad.

La bibliografía en la que se apoya este artículo, y mis propias experiencias como docente e investigadora, ${ }^{3}$ me permiten sostener que la mayoría de los proyectos escolares de ESI, que a partir de la ley se fueron desarrollando, están atravesados por un "silencio sistemático" en torno al "deseo, las emociones y los sentimientos" (Báez, Malizia y Melo, 2017: 11). Esas dimensiones, como la noción de que la ESI y la vivencia de una sexualidad plena y libre de violencias configura un derecho, quedan desdibujadas frente a la insistencia de iniciativas centradas en el modelo biomédico con énfasis en la prevención de enfermedades y embarazos; en la dimensión moralizante destinada a controlar la sexualidad teniendo como regla, entre otros criterios, la hetero-normatividad; y en las aristas judicializantes, con eje en los abusos, la trata y la violación (Díaz Villa, 2012; Kornblit, Sustas y Leo, 2014; Morgade y Fainsod, 2015; Lisboa y Sosa, 2015). Desde allí, estos estudios sostienen que en el posicionamiento institucional de la mayoría de las propuestas escolares de ESI persisten "significaciones patriarcales y hetero-normativas" (Morgade y Fainsod, 2015: 42) y, en vínculo con ello, "una imagen de la sexualidad como peligro que no solamente suele estar lejos de las experiencias juveniles sino que en muchos casos deriva en efectos contrarios a los principios que se pretende transmitir (o inculcar)" (Zattara, 2018: 10).

En relación con lo dicho, esas investigaciones sostienen que varias experiencias de ESI desarrolladas escolarmente presentan tensiones entre los currículums oficial, oculto y nulo, ${ }^{4}$ que evidencian distancias entre las intenciones, los discursos y las prácticas docentes sobre la temática, como rasgo distintivo de su efectivización en las aulas. Estos desencuentros, entre otras dimensiones, se relacionan con ciertas dificultades del colectivo de enseñantes para plantearse preguntas centradas en sus propios posicionamientos y experiencias en torno a las sexualidades (Kornblit, Sustas y Di Leo, 2014).

En este artículo describo algunas actividades de ESI desarrolladas en el nivel secundario, ${ }^{5}$ en primer lugar, de una institución educativa de gestión privada situada en un municipio del área norte de la provincia de Buenos Aires, en la que fui convocada para trabajar como orientadora, haciendo énfasis en los límites que se producen para su plena efectivización. ${ }^{6}$

Tomo como referencia metodológica para la primera parte de mi artículo la propuesta que Daniel Pennac presenta en Mal de Escuela (2008), cuando analiza ciertas dinámicas

3 Desde 2009, como magíster, doctoranda y miembro de un equipo de investigación, estudio experiencias de maternidad juvenil. En 2011 fui parte de un proyecto financiado por el Fondo de las Naciones Unidas para la Infancia (UNICEF), para evaluar propuestas de implementación de la ESI en diferentes jurisdicciones del país. En 2012 y 2013 trabajé como tallerista de ESI en propuestas de capacitación desarrolladas por el Ministerio de Educación de la Nación. Desde 2015, soy parte del equipo ESI de la Secretaría de Género de la Confederación de Trabajadores de la Educación de la República Argentina (CTERA), que también realiza capacitaciones en la materia. En 2016 dicté un seminario en la maestría de ESI de la Universidad de Cuenca (Ecuador) y empecé a enseñarla en dos institutos de formación docente de la Ciudad Autónoma de Buenos Aires. Por último, desde 2019, integro el equipo de profesoras de la Diplomatura en Formación en Educación Sexual Integral (ESI) de la Universidad Nacional de San Martín.

4 De acuerdo a la bibliografía consultada para la escritura de este artículo, el currículum oficial refiere a los contenidos que se explicitan en los programas, y el oculto a los que se transmiten de manera tácita. En su análisis de la ESI en una escuela secundaria de gestión pública de Mendoza, Micaela Lisboa y Lucía Sosa (2015: 28) sostienen que los contenidos del currículum oculto de la educación sexual aluden particularmente a los "significados transmitidos que pautan prácticas y comportamientos esperados en sexualidad" según la edad y genitalidad de lxs aprendientes. El currículum nulo, por su parte, refiere al "conjunto de contenidos que no están presentes o lo están de modo insuficiente, en las currículas oficiales, pero cuya inclusión en el proceso de enseñanza-aprendizaje constituye una demanda de los alumnos o de la sociedad" (Eisner, 1979, cit. por Kornblit, Sustas y Di Leo, 2014: 177).

5 La escuela posee nivel inicial, primario y secundario.

6 En la escuela de gestión privada que estoy describiendo aquí están al tanto de mis actividades como investigadora. Sin embargo, no he compartido las reflexiones finales que vierto en este artículo con ninguno de sus actores. Este artículo considera los resguardos éticos de rigor y los criterios de confidencialidad y anonimato. En esa línea, no menciono los nombres de personas, de instituciones ni de barrios. 
escolares partiendo de sus propias experiencias primero como estudiante y luego como profesor. Esta segunda dimensión es la que guía mis reflexiones.

A su vez, considero la crónica de Damián Huergo y Marcela Martínez (s/f) y su decisión de partir de una descripción exhaustiva de diversas situaciones que comprenden contenidos de ESI en varias escuelas, teniendo particularmente en cuenta las voces de lxs docentes que, junto a estudiantes, las protagonizan.

Luego de abordar las experiencias de Educación Sexual en el nivel secundario de la institución de gestión privada que he presenciado o protagonizado como orientadora escolar, establezco un diálogo entre las mismas y las desarrolladas en un Bachillerato Popular ubicado en un barrio del sur del conurbano bonaerense. ${ }^{7}$ Estas experiencias emergen de los primeros análisis del corpus de datos construido a lo largo del trabajo de campo de mi tesis doctoral, particularmente del relato de C., una de las jóvenes que ha participado del mismo.

Como ya he anticipado, mi tesis analiza las experiencias de escolaridad y maternidad de siete jóvenes madres y una gestante de sectores populares del Área Metropolitana de Buenos Aires, y cómo las mismas se relacionan con el vínculo construido con sus propias madres. La metodología de esta investigación es el enfoque biográfico.

Como desarrollo en este artículo, en ambas escuelas se producen conflictos a la hora de enseñar contenidos de ESI, sobre todo aquellos que hacen al respeto por la diversidad y al ejercicio de los derechos, que se anudan significativamente a los rechazos de una parte del estudiantado y sus familias en torno a los mismos. En el caso de la escuela de gestión privada, estos rechazos tienen un fundamento significativo en la fe religiosa evangélica o Testigos de Jehová que practican gran parte de ellxs. ${ }^{8}$ En el Bachillerato Popular, las controversias tienen que ver con un desconocimiento de las normativas vigentes de educación sexual, de salud sexual y reproductiva, y sobre los derechos de niños, niñas y adolescentes (leyes 26.150, 25.673 y 26.061, respectivamente). Sin embargo, dichos rechazos son tramitados de diferentes formas por las instituciones que aquí describo. Ante su emergencia, en la primera, se opta por suspender las propuestas de ESI, mientras que en la segunda, por sostenerlas.

A modo de hipótesis, interpreto que estas diferencias se relacionan, entre otras dimensiones, con la lectura institucional en torno al conflicto: o bien este se concibe como algo que hay que evitar y, en caso de producirse, anular; o bien se considera como parte de las condiciones de enseñanza.

\section{La escuela de gestión privada}

La escuela de gestión privada está ubicada en un partido de la provincia de Buenos Aires, en el norte del área denominada Aglomerado del Gran Buenos Aires. Fue fundada hace tres décadas, es laica y está subvencionada por el gobierno de esa provincia.

\footnotetext{
7 Los Bachilleratos Populares son espacios educativos destinados a jóvenes y adultos creados por organizaciones políticas con fuerte inserción barrial. Sus docentes provienen, por lo general, de instituciones universitarias. Nacen a principios de 2000 a fin de acercar la escuela secundaria a personas que por diferentes motivos (entre los que están padecer pobrezas materiales y vulnerabilidades fundamentadas en el sexo-género) se han distanciado de la misma o bien nunca la comenzaron, y se ubican fundamentalmente en las zonas más empobrecidas del Área Metropolitana de Buenos Aires. Entre las investigaciones que los estudian cito los trabajos de Nora Gluz (2013) y de Paola Rubinsztain, Shirly Said y Ana Blaustein Kappelmacher (2017).

8 El estudio de las relaciones entre las concepciones religiosas y la implementación de la ESI configura un área de vacancia (Lisboa y Sosa, 2015).
} 
Recibe chicxs que si bien pertenecen a diversos sectores sociales con distintas posibilidades económicas, lejos están de presentar dificultades para la satisfacción de necesidades básicas tales como el acceso a una alimentación nutritiva y variada, el cuidado de la salud y/o a una vivienda digna. Varios de sus padres y madres se dedican al comercio, a la docencia o a profesiones tales como la medicina o la abogacía. Muchas de esas familias, como ya mencioné, al igual que varixs docentes, practican la religión evangélica o Testigos de Jehová.

Uno de los rasgos más sobresalientes de la institución es la significativa insistencia de vínculos familiares y de amistad entre sus docentes y entre sus docentes y estudiantes. Desde allí, los apellidos se repiten una y otra vez, y varixs maestras, preceptoras y profesorxs han tenido y tienen entre sus alumnxs a sus propixs hijxs y sobrinxs. ${ }^{9}$

Esta insistencia imprime una dinámica institucional que describo como endogámica, que suele obstaculizar en muchas ocasiones el establecimiento de las distancias entre enseñantes y aprendientes necesarias para que el acto pedagógico tenga lugar. En este sentido, comento que se producen asiduamente discusiones porque una docente, por ejemplo, le ha llamado la atención al hijx de otra, o bien se abstiene de intervenir de tal o cual forma, aun juzgándolo necesario, para "no tener problemas" con quien, además de colega, es su vecinx o pariente. En esta línea, cabe mencionar que es muy raro escuchar que haya alguien en la escuela que viva en algún barrio por fuera del partido donde esta se localiza. Una de las pocas personas que presenta esa condición soy yo.

\section{Actividades de ESI en la escuela de gestión privada}

Comienzo a trabajar en la institución de gestión privada como parte de su equipo de orientación escolar, junto a la psicóloga que desde hace varios años se desempeña allí, ocupándose básicamente de tareas de derivación y seguimiento de tratamientos de chicxs que presentan dificultades de aprendizaje relacionadas con sus particularidades emocionales, psicológicas o psíquicas. ${ }^{10}$

La propuesta que me hicieron desde la dirección del nivel secundario y su departamento de asesores legales al comenzar mis tareas allí fue que, además de acompañar los procesos relacionados con dichas derivaciones y seguimientos, coordinara talleres centrados en el mejoramiento de los vínculos entre lxs chicxs de $1^{\circ}, 2^{\circ}$ y $3^{\circ}$ año de dicho nivel (luego la propuesta se extendió a $5^{\circ}$ y $6^{\circ}$ grado de la primaria).

La iniciativa estaba estrechamente relacionada con una preocupación: la significativa baja de matrícula que se había producido en los últimos años, debido a que muchxs estudiantes habían sido víctimas de violencia verbal (fundamentalmente realizada por medio de cadenas de what app) por parte de otrxs alumnxs. Desde allí, el supuesto fue que aquella merma se revertiría si la institución habilitaba un espacio para el abordaje de los conflictos subyacentes en dichas decisiones de cambio de escuela.

Mi experiencia como tallerista de ESI y mi recorrido investigando temáticas relacionadas con las sexualidades me llevaron a querer desarrollar dichos talleres considerando esa perspectiva. Para ello, conversé con mi nueva compañera de trabajo y con la vicedirectora del nivel secundario, quienes se manifestaron de acuerdo con la idea.

9 Lo mismo cabe mencionar para el personal administrativo. Por otra parte, en la escuela no hay maestros ni preceptores varones, ni personas que se identifiquen como trans.

10 En la escuela hay alumnxs que han presentado diagnósticos psiquiátricos y toman medicación de esta índole. 
Asimismo, además de los talleres mencionados, estuve involucrada en varias situaciones y actividades que tienen por centro la ESI. A continuación, presento una de ellas que tuvo lugar en un $3^{\circ}$ año, porque entiendo que ilustra con bastante claridad la virulencia que suscitan en parte del estudiantado las diversidades sexuales, y la poca capacidad por parte de la dirección del nivel para promover la reflexión en torno a ello.

En efecto, a partir de unas conversaciones que sostuve con algunas alumnas de ese curso, detecté la violencia verbal, que incluyó amenazas de golpizas, hecha de forma directa y vía what app, que sufría un alumno por haber expresado su deseo de explorar sexualidades no hetero-normativas. Dichas violencias eran ejercidas por un grupo de varones autoproclamado "homofóbico", del que participa activamente un estudiante practicante de la religión evangélica. ${ }^{11}$

En ese momento, la dirección del nivel secundario intervino con sanciones a quienes ejercieron dichas violencias, sanciones que inmediatamente se pusieron en suspenso debido a la presión realizada por parte de las familias de los jóvenes involucrados, a través del grupo de what app de padres y madres, con el apoyo de otras que se le sumaron.

En este marco, alrededor de una semana después de producirse estos hechos y con la fuerte convicción de que era necesario intervenir subrayando la necesidad de reflexionar sobre lo ocurrido, propuse desarrollar espacios de debate en torno a la temática, a partir de la proyección de un video que lleva por título Respeto por la diversidad, producido por el Programa de Educación Sexual del Ministerio de Educación. Si bien varixs estudiantes del curso en cuestión aceptaron participar de esta actividad, el grupo de alumnos autodenominado "homofóbico" mencionado anteriormente no quiso hacerlo. Comuniqué lo sucedido a la directora y vice del nivel, sin recibir respuesta alguna.

En esta propuesta consideré la puerta de entrada para el desarrollo institucional de la ESI denominada Episodios que irrumpen en la vida escolar (Ministerio de Educación de la Nación, 2010). La misma consiste básicamente en aprovechar las situaciones disruptivas que ameritan una intervención pedagógica centrada en la ESI, con el fin de habilitar debates y reflexiones que, en esta ocasión en particular, se relacionaron con valorar y respetar las diversidades.

\section{La Feria de Ciencias y la ESI}

En ocasión de una de las Feria de Ciencias que me tocó presenciar en la escuela de gestión privada, algunxs chicxs de la secundaria organizaron actividades destinadas a la primaria, centradas en el abordaje de temáticas tales como la diversidad de familias. Esto provocó discusiones de alto voltaje verbal entre varias docentes de ambos niveles, básicamente provocadas por la acusación que algunas maestras hicieron con relación a esas actividades, subrayando lo que consideraron como un "contenido pornográfico" totalmente desaconsejable para la edad de sus alumnxs.

Este conflicto condujo a la asesoría legal del establecimiento y al equipo de orientación a plantear dos líneas de trabajo. Por un lado, potenciar la comunicación a través de

11 La forma en que logro detectar estas situaciones de violencia pueden ser puestas en diálogo con el emergente Secreto que propone Molina (2015) en sus reflexiones sobre escuelas y sexualidades adolescentes en Córdoba, a partir de un trabajo de campo realizado entre 2004 y 2010. El deseo del muchacho que cito aquí por explorar una sexualidad no hetero-normativa es compartido, primero, en su grupo cercano de amigas, para luego, "no se sabe cómo", comenzar a circular entre los varones autodenominados homofóbicos. A partir de allí, empiezan las agresiones y amenazas comentadas, de las que tomo conocimiento por medio de esas chicas. Interpreto que este grupo opera como resguardo ante esas hostilidades y violencias, al mismo tiempo que se configura como un espacio de confianza en donde es posible compartir el secreto. 
reuniones periódicas entre la primaria y la secundaria a fin de que merme la posibilidad de malos entendidos cuando de desarrollar la ESI se trate. Por otro, la necesidad de motorizar instancias de capacitación en la materia, destinadas al conjunto de docentes de nivel inicial y primario, dado que se interpretó que el secundario, en este punto, contaba con mayores herramientas. Con relación a la primera línea, desde que fue planteada hasta el momento de escritura de este artículo, no ha presentado avances. En relación con la segunda, por el contrario, sí los hubo, aunque, como describo a continuación, con importantes límites.

En efecto, con la colaboración de la psicóloga de la escuela, planifiqué un taller a desarrollar en dos instancias, una al finalizar el ciclo escolar que se cerraba tras la Feria, y otra al comenzar el ciclo siguiente, durante el periodo posterior y previo al inicio de clases respectivamente, cuando el colectivo docente tiene la obligación de asistir a la escuela, pero no está con alumnxs a cargo.

La primera instancia del taller se centró en el marco legal de la ESI. Tuvo buena recepción y habilitó el desarrollo de debates muy enriquecedores que aclararon algunas cuestiones básicas como la existencia, desde hace más de una década, de una normativa que obliga a enseñarla en todas las escuelas. ${ }^{12}$ La segunda instancia tuvo como propósito darle lugar a la Pregunta por lo que nos pasa en relación a las sexualidades, una de las puertas de entrada que permite su abordaje institucional (Ministerio de Educación de la Nación, 2010).

La Pregunta por lo que nos pasa es un interrogante relacionado con lo que sentimos, pensamos y practicamos en torno a las sexualidades, que debe formularse tanto individual como institucionalmente, dada nuestra condición de seres sexuadxs. Desde allí, se entiende que la docencia es una de las tantas particularidades que nos configura como sujetxs, que va unida y está atravesada por otras dimensiones biográficas tales como los vínculos que sostenemos con otrxs, nuestras maneras de pensar y de sentir, nuestras prácticas y emociones, y nuestras inscripciones políticas, sociales, culturales y religiosas. Habilitar la pregunta por lo que nos pasa en torno a las sexualidades implica un trabajo de visualización, desnaturalización y re-significación de nuestras experiencias (Kornblit, Sustas y Leo, 2014), insoslayable para que dichas particularidades no devengan obstáculos para el despliegue de la ESI.

Esta segunda línea de trabajo fue suspendida 48 hs. antes de llevarla a cabo, por las directoras del nivel inicial y primario, bajo el argumento de que les faltaba tiempo para preparar la escenografía que requería el acto de inicio del ciclo lectivo que comenzaba unos días después.

Lo descripto aquí, desde mi perspectiva, da pistas sobre el posicionamiento de la escuela en torno a la ESI, fundamentalmente con relación a los obstáculos que se producen cuando se trata de explorar en las propias miradas sobre las sexualidades y explicitar entre colegas nuestros miedos, dudas y pre-nociones, con el propósito de llegar a acuerdos para abordarla institucionalmente. Como dije, desde que fueron planteadas estas dos líneas de trabajo hasta el momento de escritura de este artículo, no fue posible establecer espacios de diálogos entre niveles, así como tampoco profundizar el ejercicio de la pregunta por lo que nos pasa. ${ }^{13}$ 


\section{Videos, quejas y acusaciones}

Al comenzar el siguiente año, se planteó una novedad en el nivel secundario de la escuela de gestión privada: la realización de una semana de la ESI hacia la mitad del ciclo lectivo, destinada a abordar específicamente la temática a lo largo de cinco días consecutivos.

A raíz de esta propuesta, conversé con la psicóloga de la escuela y la vicedirectora del nivel, haciéndoles saber que deseaba preparar actividades con lxs chicxs de los años $1^{\circ}, 2^{\circ}$ y $3^{\circ}$ para participar de dichas jornadas, aprovechando el espacio que brindan los talleres mencionados anteriormente. Estuvieron de acuerdo. Consensuamos un modo de trabajo que partiera de sus intereses. Desde allí, la idea fue que lxs estudiantes decidieran qué temáticas específicas iban a abordar y los modos de hacerlo.

De esta manera, planteé la propuesta en los cursos. Según entendí, la misma, en términos generales, fue aceptada. Sin embargo, los hechos que sucedieron luego en un curso de $3^{\circ}$, diferente al mencionado anteriormente, me hacen pensar que evalué mal esta primera predisposición.

En un primer encuentro les comenté a lxs chicxs de ese curso cuáles eran los lineamientos generales de la propuesta, haciendo énfasis en la idea de que ellxs iban a ser lxs que decidieran qué temas específicos se iban a tocar y cómo lo dicho se pondría en práctica. Me pareció importante partir, entonces, de una instancia en donde se desarrollara la dinámica conocida como "lluvia de ideas". Mediante la misma, lxs estudiantes irían proponiendo temáticas y modalidades de abordaje que se consignarían en el pizarrón, en sus carpetas y/o en mis registros, con el fin de llegar a acuerdos para motorizar la idea. Mi intención inicial fue destinar a este ejercicio como mínimo tres encuentros, con posibilidades de extender la dinámica si se presentara como necesario.

La charla que se produjo luego de esta presentación, sobre todo con algunas alumnas que suelen manifestarse especialmente interesadas en la temática, si bien fue y vino por varios tópicos inherentes a la ESI, derivó en un comentario en torno a un video visto en una clase con otra profesora que abordó el consentimiento en torno a tener relaciones sexuales haciendo un juego con la idea de invitar a alguien a tomar el té.

Recordé un video de origen paraguayo bastante parecido que un colega de otra institución me había mostrado, pero que, en vez de té, metaforizaba dicho consentimiento considerando la bebida conocida como tereré. Como me pareció interesante compartirlo, les comenté a lxs chicxs que, en el próximo encuentro lo proyectaría, con la intención de empezar a hacer circular materiales que, a partir de la dinámica "lluvia de ideas" antes mencionada, les diera pistas para desarrollar sus propias propuestas.

Al terminar este primer encuentro, se me acerca la alumna M. para decirme, de manera privada, que no quiere participar de la propuesta y que su decisión se relaciona con su religión Testigos de Jehová. Le respondí que respetaba su postura (estoy absolutamente convencida de que la coerción no es un camino recomendable en este ni en ningún caso), pero que, a la vez, necesitaba decirle que no me parecía buena idea que se apartara. Por el contrario, lo mejor, desde mi perspectiva, era que participara dando cuenta de sus convicciones, aceptando abrir un debate con sus compañerxs.

$\mathrm{Al}$ encuentro siguiente, llevé el citado video sobre el consentimiento. Luego de la proyección se establecieron puentes con el material visto en la otra materia y, por pedido del grupo de chicas más entusiasta con la ESI ya mencionado, proyecté varios videos más. Mi propósito siempre fue que circularan materiales que permitieran seguir indagando qué temáticas específicas podrían ser tratadas en las jornadas y bajo qué modalidades. 
En esta línea, propuse ver un material que me había llegado a través de un investigador vinculado a una escuela confesional ubicada en la zona oeste del Gran Buenos Aires. ${ }^{14}$ El mismo narra la historia de amor entre dos chicas. Lo traje a colación no tanto por la temática en sí, sino por la forma en que esta se aborda: una animación tipo stopmotion, de no más de cinco minutos, que tiene como música de fondo una canción de estilo pop, que suele escucharse por las radios. Luego, propuse escuchar la canción "Costurera carpintero" del cantautor Gabo Ferro, sobre un niño costurera y una niña carpintero, y "Eva” de Silvio Rodríguez.

Alterné la proyección de estos vídeos y la escucha de estas canciones con otras propuestas que fueron haciendo algunas de las chicas más entusiastas con trabajar la ESI: un video en el que aparece el locutor Lalo Mir describiendo en qué consiste este campo de conocimientos; otro que desarrolla algunos ejes claves de la perspectiva de género y muestra escenas de las manifestaciones que acompañaron el tratamiento en el Congreso del proyecto de ley para legalizar la interrupción voluntaria del embarazo; y, por último, otro que pone en tensión los estereotipos de belleza que suelen producirse y reproducirse a través de las películas de Disney.

M. no participó en ningún momento del encuentro. Pero, al finalizarlo, se me acerca y, una vez más de manera privada, me entrega una tarjeta en la que figura una dirección de sitio web. Allí, me dice, puedo conocer de qué se trata su religión. Asimismo, vuelve a comentarme que no quiere participar de la propuesta.

Mientras guardaba entre mis pertenencias la tarjeta, insistí en la idea de que, para amortiguar cualquier tipo de incomodidad que se pueda producir, lo mejor era que preparase una contra-argumentación para el próximo encuentro, y/o trajese el material que sí le parecía mejor ver. M. mantuvo su negativa.

A los tres días de lo narrado, la directora de nivel secundario me comenta que la madre de M. se quejó ante ella por el tratamiento que en torno a la ESI estoy desarrollando. El eje de dicha queja era, básicamente, que yo "les decía a los chicos que se puede estar hoy con uno, con otro mañana y otro pasado, porque total, después se puede abortar" y que "mostraba videos en donde tenían sexo dos varones".

Me sentí muy molesta con la situación, porque, básicamente, la viví como una acusación falsa. Sin embargo, acordamos con la directora que lo mejor era que conversara con la madre para aclarar qué había sucedido. De este modo, la cité, vía un llamado telefónico que hizo una preceptora, para una reunión que tuvo lugar un par de horas después.

Dicha reunión, realizada solo entre la madre y yo, comenzó en muy malos términos. Porque, además de insistir con la idea de que mis propuestas promueven la promiscuidad, comentó que la queja se fundamentaba en algo que "habían dicho los chicos" el año anterior. Con ese fundamento, entre otras cuestiones, objetó el vocabulario utilizado por mí en los talleres dado que lo consideraba inapropiado para ser escuchado por adolescentes de 14 años; ${ }^{15}$ me comentó que estaba de acuerdo con la ESI en tanto y en cuanto la misma se desarrollase "como lo hace la profesora de biología", pero no como yo propongo; me advirtió que tenía que tener cuidado con lo que decía porque lxs chicxs a esa edad no tienen relaciones sexuales y podía promover que las tuvieran; y subrayó la necesidad de que su hija estuviese tan cómoda en todas las instancias y propuestas de la escuela como lo está en su casa. 
Una vez pasado el primer impacto del malestar (imposible de disimular, por cierto) que se apoderó de mí al escucharla, le expliqué que mis intervenciones estaban fundamentadas en la Ley de ESI y sus lineamientos curriculares, y que los materiales que utilizaba eran los propuestos por el Ministerio de Educación de la Nación, me habían sido recomendados por colegas y/o eran propuestas que traían lxs mismxs estudiantes.

A partir allí, hice hincapié en lo ocurrido en los últimos encuentros descriptos anteriormente y en la idea de que su hija estaba absolutamente habilitada para proponer otros materiales y/o tomar la palabra. Pero, tal como hizo M., su madre desestimó esta idea.

Me parece importante añadir que no es la primera vez que me veo interpelada por estudiantes pertenecientes a la fe Testigos de Jehová y otras religiones, cuando desarrollo los talleres de ESI. Lo que es inédito para mí es el nivel de violencia que acompañó, en esta ocasión, dicha interpelación. En este sentido, entiendo que las incomodidades que pueden producirse a partir del encuentro entre los contenidos que enseño y las pertenencias religiosas de mis estudiantes son parte de mi trabajo y que la misma ESI ofrece una herramienta muy potente para tramitarlas: la ya descripta puerta de entrada, centrada en la pregunta por lo que nos pasa con relación a las sexualidades. Este interrogante, de considerarse necesario, puede plantearse también en un taller destinado a familias.

Pasado el primer impacto, y en un tono más tranquilo, le propuse ver a la madre de $\mathrm{M}$. los vídeos compartidos con su curso, a fin de que chequeara por sí misma que nada de lo que circuló tuvo que ver con escenas en donde, por ejemplo, dos varones tenían sexo. Lo dicho se frustró de inmediato porque el medio tecnológico con el que contaba en ese momento (una computadora portátil de la escuela) no funcionó.

Al finalizar la reunión, creí visualizar en esa madre cierta intención de irse en mejores términos de los que había llegado. Pronto me di cuenta de que estaba equivocada.

Efectivamente, a los pocos días se desarrolló la primera reunión anual de familias de ese $3^{\circ}$ año. Algunas de ellas manifestaron allí su disconformidad en torno a mis propuestas con la directora, en la misma línea que la madre de M., quien insistió con su tesitura, aunque, nuevamente, lo hizo de manera privada.

A partir de allí, tanto la directora como la vice del nivel me llaman la atención sosteniendo que hay que dejar de realizar los talleres desde la perspectiva que deseo imprimirles.

\section{El Bachillerato Popular: el barrio, sus alumnxs y docentes}

Lo descripto, considerando mi labor como orientadora escolar, contrasta con lo que emergió en el marco del trabajo de campo de mi tesis doctoral.

Se trata de una investigación cualitativa preocupada por las escolaridades secundarias de ocho jóvenes vulnerabilizadas del Área Metropolitana de Buenos Aires con experiencias de maternidad y los vínculos que sostienen con sus propias madres. Su metodología, como ya he anticipado, es el enfoque biográfico. ${ }^{16}$

16 Brevemente, este enfoque propone la construcción de relatos con las personas que participan de una investigación, vertebrándolos con sus acontecimientos biográficos claves, a partir de la realización de varias entrevistas en profundidad. Estos acontecimientos son hechos que, marcando un antes y un después en las vidas de esas personas, operan como experiencias desde las cuales parte el análisis (Ferrarotti, 2007; Leclerc, 2009; Di Leo y Camarotti, 2012). Por lo demás, como mencioné con relación a la escuela de gestión privada, al referirme al Bachillerato Popular considero los resguardos éticos de rigor y respeto los criterios de confidencialidad y anonimato. Desde allí, no nombro personas, instituciones ni barrios. 
En este marco, entre 2015 y 2017 construí diferentes relatos biográficos con siete mujeres madres y una gestante, de entre 18 y 24 años, cuatro de sus madres y diez de sus docentes, a través de la elaboración de varias entrevistas en profundidad, el análisis de objetos personales tales como fotos, informes médicos, carpetas y boletines escolares, y la visita a sus barrios y escuelas.

Como dije al comenzar este artículo, una de las chicas que participaron de mi investigación es C., una joven de 24 años, madre de una nena de 7, y alumna de un Bachillerato Popular que se ubica en el área sur de la provincia de Buenos Aires, en un municipio del segundo cordón del conurbano. Las reflexiones que propongo a continuación se basan en sus entrevistas, en las que sostuve con su madre y sus docentes, y en las notas de campo que realicé en cada uno de nuestros encuentros.

El Bachillerato Popular en el que C. estudia comparte algunas características con la otra escuela aquí descripta: es laica, se sitúa en la provincia de Buenos Aires, y la mayoría de sus estudiantes (por no decir la totalidad) son vecinxs del barrio. Sin embargo, al contrario de aquella, esos públicos tienen diferentes edades, viven en condiciones de vulnerabilidad material, y han atravesado experiencias de alejamiento de la escuela secundaria antes de su ingreso a la institución. A su vez, esta fue creada en un momento posterior a la de gestión privada, como parte de un proyecto de militancia política con fuerte inserción territorial.

La mayoría de las casas del barrio donde se ubica la escuela presentan situaciones de precariedad (entre otras, hacinamiento, problemas de tipo edilicio y falta de cloacas). Las calles son de tierra y está atravesado por un arroyo contaminado. Varixs de lxs vecinxs, entre quienes están lxs propixs alumnxs, tienen dificultades laborales (trabajan de manera intermitente y en empleos que exigen mucho esfuerzo físico, que se desarrollan por fuera de la normativa vigente) y reciben, por ejemplo, la Asignación Universal por Hijo. ${ }^{17}$

Lxs docentes de este Bachillerato Popular no viven en el vecindario, tienen experiencias de militancia más desarrolladas en el tiempo que algunxs de sus estudiantes, pertenecen a sectores socioeconómicos mejor posicionados que ellxs, cuentan con estudios terciarios o universitarios, y no están unidxs familiarmente a sus alumnxs. De este modo, la institución no presenta la dinámica endogámica que sí visualicé en la escuela de gestión privada.

Por último, otra característica que diferencia al Bachillerato Popular de la escuela de gestión privada es que la religión no tiene un tipo de importancia particular para sus docentes y la comunidad.

A partir de esta breve descripción general de la institución, a continuación abordo las tensiones que se produjeron en el marco de un taller de ESI.

\section{La ESI y el ejercicio de los derechos sexuales y reproductivos}

Una de las experiencias que C. más disfruta de su escolaridad son los talleres de ESI que coordina A., una docente-médica. A continuación, recorto de una de sus entrevistas el siguiente emergente: 
Porque A. trabaja en el Argerich ${ }^{18}$ [...]. Hoy en el Argerich están dando número para el nuevo método del catéter en el brazo, que larga hormonas [...] nos ofreció, porque era un método caro y que se estaba dando gratis en el hospital, para que vayamos y pidamos turnos para que aprovechemos esto porque es caro y el Estado dio solamente stock a algunos lugares. Y nos dio una mano para que vayamos y lo podamos utilizar. Pero, a eso voy. La información... Porque nos abrió la cabeza en un montón de cosas... En decir, por ejemplo, tengo una hermana que es lesbiana, encuentra deseo en una mujer. Y yo le hablo un montón. Porque a mí me dice [mi profesora] que... O sea, tu ginecólogo te tiene que preguntar todo. Vos tenés que abrirte con tu ginecólogo, en el respeto y en lo profesional, para que él te dé opciones. Porque, por más que tengas una relación con una mujer, o hombre con hombre, hay enfermedades igual. A eso voy. Hay que estar informado. Y que te den las herramientas para estar informado. $Y$, si no la tenés, tenés que denunciarlo. Es legal que haya. $\mathrm{O}$ sea, si en el hospital no lo están teniendo, está en falta. No sos vos la que está mal. Está mal la institución. $\mathrm{O}$ sea, a eso voy. Está buenísimo, ella es doctora. Y... (risas) Imaginate. Viene y nos da clase de todo. Y nos aviva en un montón de cosas de decir... eh... "vayan y pregunten, porque esto es legal, o esto es ilegal... Y, cuando ustedes se sientan, tienen que darles esta información... eh... Los doctores les tienen que dar, les tienen que brindar esta información... Tienen que pedirla... Hay un número telefónico, tienen que denunciar”... eh... todo ese tipo de información.

Desde mi lectura, esta descripción de la información que la docente brinda muestra el énfasis que da a la idea de ejercicio de los derechos sexuales haciendo hincapié en el cuidado de la salud, y a las vicisitudes que suscita dicho ejercicio.

Desde allí, dando cuenta de las distancias existentes entre las leyes y las prácticas concretas que contribuyen o no al despliegue de los derechos que aquellas garantizan, la docente explica que se pueden utilizar métodos anticonceptivos como el parche hormonal, o recibir tal o cual información, al tiempo que enfatiza la idea de exigir lo dicho cuando se es negado. A su vez, hace referencia a la responsabilidad en el cuidado de la salud sexual que les cabe a las instituciones sanitarias, y a los procedimientos existentes para denunciar, cuando esta no se cumple.

Dicha mirada en torno al ejercicio de los derechos es recibida de manera diferente por el conjunto de alumnas de la institución. Como presento a continuación, el posicionamiento receptivo de C. (que se expresa en palabras tales como "A. nos abre la cabeza en un montón de cosas") no es compartido por varias de las estudiantes mayores que tienen hijas adolescentes.

[A. nos dijo que] cuando vos vas a una cosa ginecológica, si vos sos menor, vos, igual, tenés derecho a elegir si querés que tu mamá esté o te espere afuera. Tu mamá no puede decir: "ella es menor, yo me quedo acá". No [...]. A. ha discutido con mamás de ahí. Porque ahí hay mamás, también. Y dicen: "no, porque mi hija..." [Como si A. les contestara a esas mujeres alumnas] “No, respeto ante todo. Tu hija no tiene por qué... Tu hija, si no quiere contártelo... que tiene relaciones con el novio, o que es activa sexual de alguna forma, no tiene por qué contártelo. Vos, como madre, tenés que tratar de incentivarla a que ella tenga las propias herramientas como para poder autocuidarse".

Estas discusiones, desde mi perspectiva, expresan un desconocimiento por parte de esas mujeres adultas estudiantes del Bachillerato Popular sobre las leyes 26.150, 25.673 y 26.061, y los derechos que las mismas sancionan. 
Ahora bien, aunque emerjan conflictos con parte de esas alumnas, lejos se está de suspender la propuesta de ESI con eje en el ejercicio de los derechos, teniendo como fundamento la incomodidad que su tratamiento produce. Por el contrario, como se desprende de lo dicho, e insiste en las conversaciones que he sostenido con C. sobre el tema, se trata menos de obstaculizar la discusión que de darle lugar.

Desde allí, encuentro que la decisión de sostener la enseñanza de estos contenidos se relaciona con una dimensión del posicionamiento institucional del Bachillerato Popular en torno a la ESI, ausente en la escuela de gestión privada: la aceptación del alumnado de los mismos no es un punto de partida necesario para su abordaje, sino que, por el contrario, deviene un punto de llegada posible, o no, del proceso pedagógico.

\section{Reflexiones finales}

Las descripciones hechas en este artículo se emparentan con los estudios que analizan el devenir escolar de la ESI desde la sanción de la Ley 26.150.

Desde allí, exploro experiencias ocurridas en la escuela de gestión privada, dando cuenta, por ejemplo, de las vicisitudes que acarrea el desarrollo de propuestas centradas en el respeto por las diversidades. A su vez, hago visible una dificultad para el armado de espacios institucionales destinados a la pregunta por los que nos pasa en torno a las sexualidades, difícil de tensionar dada la voluntad de los equipos directivos de, por acción u omisión, no permitirlos. Estas dimensiones, al articularse entre sí, obstaculizan el abordaje pleno de la ESI, dando cuenta de las particularidades que se presentan a la hora de efectivizar derechos sexuales sancionados legalmente.

Dichas particularidades en esa escuela se relacionan, entre otros aspectos, con los rechazos que varixs de sus estudiantes y familias practicantes de la fe evangélica o Testigo de Jehová oponen. Los mismos son avalados institucionalmente por la directora y vice de la secundaria. Desde allí, escuchan sus reclamos y quejas, y actúan suspendiendo acciones que provocan conflictos.

Esta forma de abordar las sexualidades se distancia del posicionamiento del Bachillerato Popular sobre el particular. Como he dicho, en esta institución se producen rechazos por parte de un grupo de alumnas a la hora de abordar específicamente una dimensión central de la ESI como es el ejercicio de los derechos sexuales. Sin embargo, lejos se está de suspender el tratamiento de estos temas. Desde mi perspectiva, esto se relaciona con una lectura institucional en torno a los conflictos, donde estos son parte de las condiciones de enseñanza, antes que situaciones a anular.

Entiendo que este posicionamiento con relación a la ESI, así como el vínculo entre su implementación y las concepciones religiosas de sus docentes y estudiantes, o la articulación entre su enseñanza y el carácter endogámico institucional, configuran dimensiones a seguir explorando teórica y pedagógicamente.

Dicha propuesta, junto a la organización de espacios institucionales para habilitar la pregunta por lo que nos pasa en torno a las sexualidades, destinados a docentes, alumnxs y familias, puede contribuir a poner en tensión los obstáculos mencionados y, desde allí, potenciar el desarrollo de la ESI. 


\section{Q Bibliografía}

》 Báez, J.; Malizia, A. y Melo, M. (2017). “Generizando” la lengua y la literatura desde la cotidianidad del aula. Rosario: Homo Sapiens.

» Di Leo, P. y Camarotti, A. (Ed.) (2012). Quiero escribir mi historia. Vidas de jóvenes en barrios populares. Buenos Aires: Biblos.

»Díaz Villa, G. (2012). Una pedagogía para la ESI (Educación Sexual Integral. Ley 26.150/06). Cuadernos de educación, X (10), s/d.

» Faur, E.; Gogna, M. y Binstock, G. (2015). La educación sexual integral en la Argentina. Balances y desafios de la implementación de la ley. Buenos Aires: Ministerio de Educación de la Nación.

"Ferrarotti, F. (2007). Las historias de vida como método. Revista UAEM, 44, pp. 15-40.

" Gluz, N. (2013). Las luchas populares por el derecho a la educación: experiencias educativas de movimientos sociales. Buenos Aires: CLACSO.

"González del Cerro, C. y Busca, M. (2017). Más allá del sistema reproductor. Aportes para la enseñanza de la biología desde la perspectiva de género. Rosario: Homo Sapiens.

» Huergo, D. y Martínez, M. L. (s/f). El aula global y el maestro moderador. Anfibia. Recuperado de <http://revistaanfibia.com/cronica/aula-global-maestromoderador/>.

» Kornblit, A.; Sustas, S. y Di Leo, P. (2014). Género, derechos sexuales, biografía y escuela: articulaciones y tensiones en discursos de docentes de Argentina. Educ. Soc., Campinas, 35 (126), pp. 161-178.

»Leclerc-Olive, M. (2009). Temporalidades de la experiencia: las biografías y sus Acontecimientos. Iberofórum. Revista de Ciencias Sociales de la Universidad Iberoamericana, 8 (6), pp. 1-39.

»Lisboa, M. y Sosa, L. (2015). Regulando las relaciones sexuales adolescentes: moral y doctrina católica en las perspectivas docentes sobre la educación sexual integral. Revista del IICE, 38, pp. 25-37.

»Ministerio de Educación (2010). Educación Sexual Integral para la Educación Secundaria. Contenidos y propuestas para el aula. Buenos Aires.

» Morgade, G. y Fainsod, P. (2015). Convergencias y divergencias de sentido en los talleres de Educación Sexual Integral de la formación docente. Revista del IICE, 38, pp. 39-62.

»Molina, G. (2015). Escuela y sexualidades adolescentes. Aportes desde la perspectiva socioantropológica. Revista del IICE, 38, pp. 75-87.

»Pennac, D. (2008). Mal de Escuela. Barcelona: Literatura Mondadori.

»Rubinsztain, P.; Said, S.; Blaustein Kappelmacher, A. (2017). Educación Pública Popular y Autonomía: Las complejas relaciones entre los Bachilleratos Populares y el Estado en Argentina. Miradas desde los propios movimientos sociales (Informe final). Buenos Aires: CLACSO.

»Zattara, S. (2018). Formación ética y ciudadana con justicia de género e igualdad social. Rosario: Homo Sapiens. 


\section{Videos}

»Programa de Educación Sexual Integral, Canal Encuentro y Ministerio de Educación de la Nación (Productores). (2010). Educación Sexual Integral. Respeto por la diversidad. Recuperado de <https://www.youtube.com/watch?v=5HazzL7vcWU>.

»Virmani, Anita (2016). Una lección en consentimiento. Recuperado de <https:// www.youtube.com/watch?v=sTgRsoeLaoY>.

" Circo Super Pop (Productores) (2018). Concierto de Palabras: Educación Sexual. Recuperado de $<$ https://www.youtube.com/watch?v=aehHk-37Rgg >.

»Canal de Mora (Productores) (2018). Libre. Mora Navarro. Recuperado de <https:// www.youtube.com/watch?v=qLBZtBySq6E $>$.

»Vicu Villanueva (Productora) (2014). Disney Miente. Recuperado de <https://www. youtube.com/watch?v=kAGkQ_GpySo>.

\section{María Soledad Vázquez}

Magíster en Ciencias Sociales con mención en Educación, Facultad Latinoamericana de Ciencias Sociales (Argentina). Licenciada y Profesora en Sociología, Universidad de Buenos Aires. Doctoranda en Ciencias Sociales, Universidad de Buenos Aires. vazquezmariasoledad1974@gmail.com 
